\title{
INFRARED ASTRONOMY
}

FRANK J. Low

(Rice University, Houston, Texas, and University of Arizona, Tucson, Ariz., U.S.A.)

The Earth's atmosphere transmits infrared radiation through a number of windows. Table 1 lists the seven photometric systems in use at the University of Arizona which are chosen to fit the windows between 1.0 and 25 microns. An absolute calibration (Johnson, 1965; Low, 1966) has been worked out for each wavelength band and, for reference, we include an estimate of our current limiting magnitudes using a 60-inch telescope. At about 1000 microns, observations from the ground are again possible and both our group and workers in Russia (Fedoseev 1963) and England (Baldock et al., 1965) have succeeded in making observations of celestial sources. Between 25 and 1000 microns a few data have now been obtained from stratospheric altitudes by observers using jet aircraft (Low and Gillespie, 1968) and helium-filled balloons (Hoffman et al., 1967). We can anticipate that activity of this sort will increase greatly in the near future. At present, however, most of what we know concerning the nature of celestial objects at infrared wavelengths was obtained with ground-based instruments.

The planets radiate strongly in the infrared and have been studied extensively. The ultra-high resolution near-infrared spectra of Connes and co-workers (Connes et al., 1967) show what can be done from the ground despite telluric contamination. Kuiper (personal communication) has recently succeeded in obtaining lower resolution spectra of Venus and Mars from a jet aircraft, with much less telluric interference. In collaboration with Stein and Gillett at the University of California, San Diego, we

Table 1

Infrared photometric systems in use at the University of Arizona

$\begin{array}{cc}\begin{array}{c}\text { Photometric } \\ \text { Designation }\end{array} & \begin{array}{r}\lambda_{\text {eff }} \\ (\mu)\end{array} \\ & \\ \text { J } & 1.25 \\ \text { H } & 1 \cdot 60 \\ \text { K } & 2 \cdot 2 \\ \text { L } & 3 \cdot 4 \\ \text { M } & 5 \cdot 0 \\ \text { N } & 10 \cdot 2 \\ \text { Q } & 22 \cdot 0\end{array}$

\begin{tabular}{lcc}
\multicolumn{2}{c}{ Absolute Flux, Mag $=0$} & $\begin{array}{c}\text { Limiting } \\
\text { Magnitude }\end{array}$ \\
$W / \mathrm{cm}^{2} / \mu$ & $W / \mathrm{m}^{2} / \mathrm{Hz}$ & \\
$3.4 \times 10^{-13}$ & $1.77 \times 10^{-23}$ & - \\
$1.28 \times 10^{-13}$ & $1.09 \times 10^{-23}$ & 13.5 \\
$3.9 \times 10^{-14}$ & $6 \cdot 3 \times 10^{-24}$ & $10 \cdot 0$ \\
$8.1 \times 10^{-15}$ & $3 \cdot 1 \times 10^{-24}$ & $8 \cdot 0$ \\
$2.2 \times 10^{-15}$ & $1.8 \times 10^{-24}$ & 5.0 \\
$1.23 \times 10^{-16}$ & $4.3 \times 10^{-25}$ & $2 \cdot 0$ \\
$7 \cdot 7 \times 10^{-18 *}$ & $1.02 \times 10^{-25 *}$ & $0 \cdot 0$
\end{tabular}

* Provisional.

Perek (ed.), Highlights of Astronomy, 136-147. (C I.A.U. 
have made absolute spectral scans of the planets from $2 \cdot 8$ to 15 microns on the ground, using the 60-inch infrared telescope at Arizona. Figure 1 shows a scan of Jupiter revealing the existence of a window at 5 microns in the Jovian atmosphere. The brightness temperature at the centre of this window is about $200^{\circ} \mathrm{K}$, corresponding to the temperature at the cloud deck. High-angular resolution $\left(\sim 4^{\prime \prime}\right)$ scans of the Jovian disk at 10 and 22 microns were made with the 61-inch planetary telescope at Arizona, showing limb darkening and meteorological features (Figure 2). The Caltech group

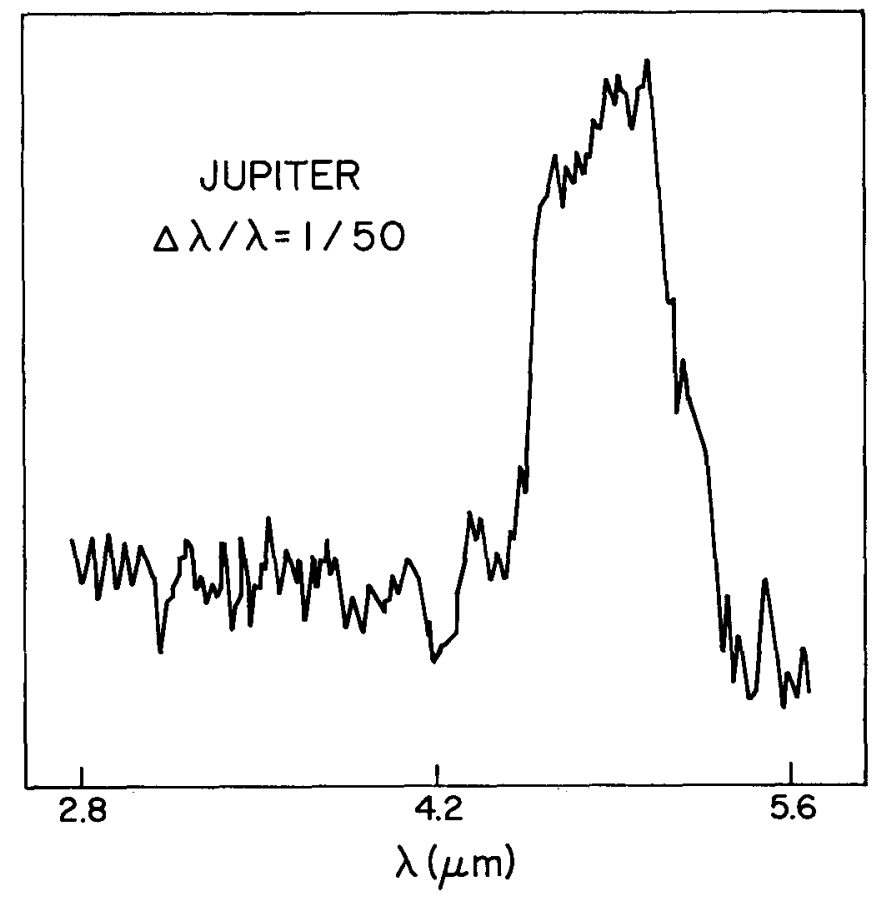

FIG. 1. Spectral scan of Jupiter, $2.8-5.6$ microns with resolution, $\Delta \lambda / \lambda, 1 / 50$. Note the relative absence of energy at 3.4 microns.

(Westphal et al., 1965) made similar studies of Venus at 10 microns, using the 200-inch telescope on Palomar Mountain. They also made interesting 1-10 micron studies of the recent comet Ikeya-Seki (Becklin and Westphal, 1966).

Turning from the planets to the Sun, we can report progress on several efforts to define the Sun's spectral energy distribution. Figure 3 shows the brightness temperature, $T_{\mathrm{B}}$, plotted against $\lambda$ from the radio region to 10 microns. Robert Noyes of the Smithsonian Astrophysical Observatory, Jacque Beckers of Sacramento Peak Observatory, and the author observed the solar limb intensity at 22 microns during the total eclipse of November 12, 1966. Center-to-limb studies were carried out by Pierre Léna, using the 60-inch solar telescope at Kitt Peak National Observatory. These 


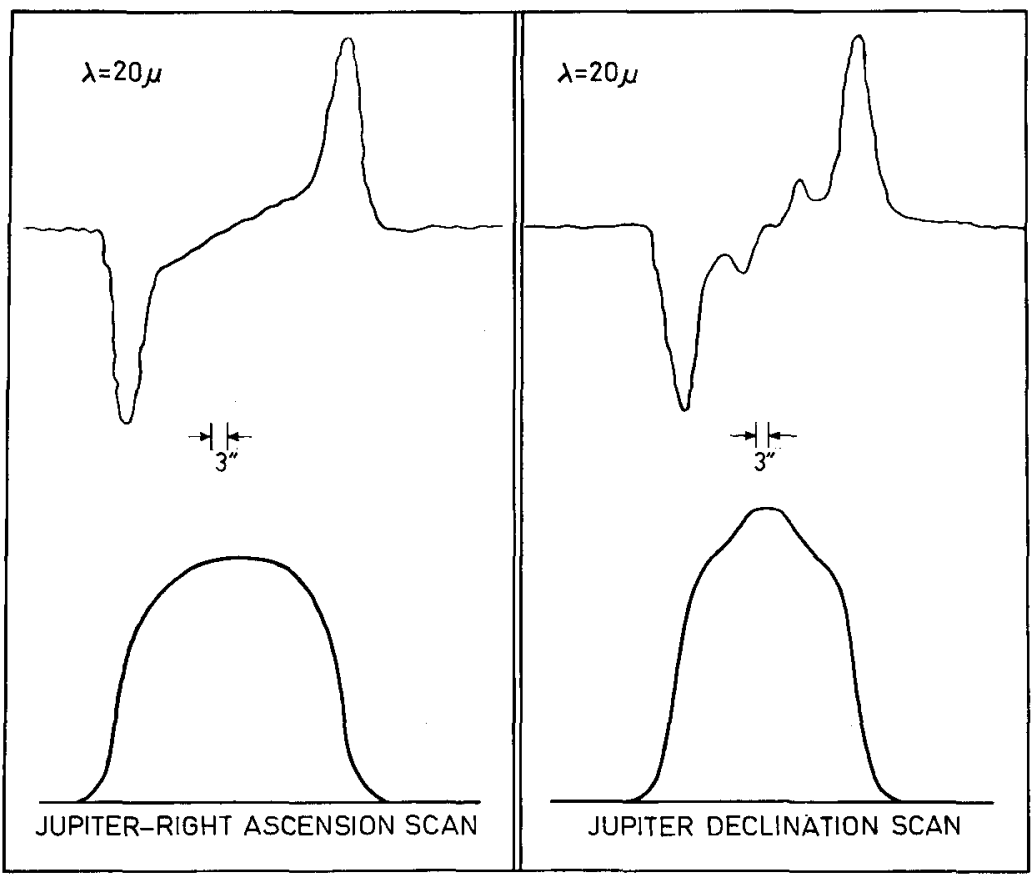

FIG. 2. Scans of Jupiter at 22 microns in right ascension and declination with an angular resolution of $4 \mathrm{sec}$ of arc.

results show that the minimum in the $T_{\mathrm{B}}$ versus $\lambda$ curve has the shape from 10 to 100 microns as shown in Figure 3. The author (Low and Gillespie, 1968) has carried out a series of absolute radiometry experiments on the Sun from an altitude of 44000 feet, in a jet aircraft. The brightness temperature at 1000 microns is in good agreement with the earlier value (Low and Davidson, 1965) obtained from the ground, when indirect corrections were made for atmospheric absorption (Low and Davidson).

Johnson and co-workers (Johnson et al., 1966) have published catalogues giving magnitudes of numerous bright stars in the range 0.3-3.4 microns. Several papers have been published by the Arizona group giving results for 5, 10, and 22 microns (Johnson and Mitchell, 1963; Low and Johnson, 1964; Low, 1966). Bolometric corrections and effective temperatures of cool stars have been obtained by use of the intrinsic colors which are now available (Johnson, 1966). Detailed studies of the spectral energy distribution of stars are of great interest both for understanding the intrinsic properties of stars and for studying interstellar and circumstellar phenomena. Figure 4 shows the absolute spectral energy curve for $\alpha$ Orionis. Note the dip at 5 microns and the excess at 22 microns.

Johnson and co-workers at Arizona (Johnson, 1965, 1968) have carried out extensive studies of interstellar extinction. Figure 5 shows the reddening law they found for 


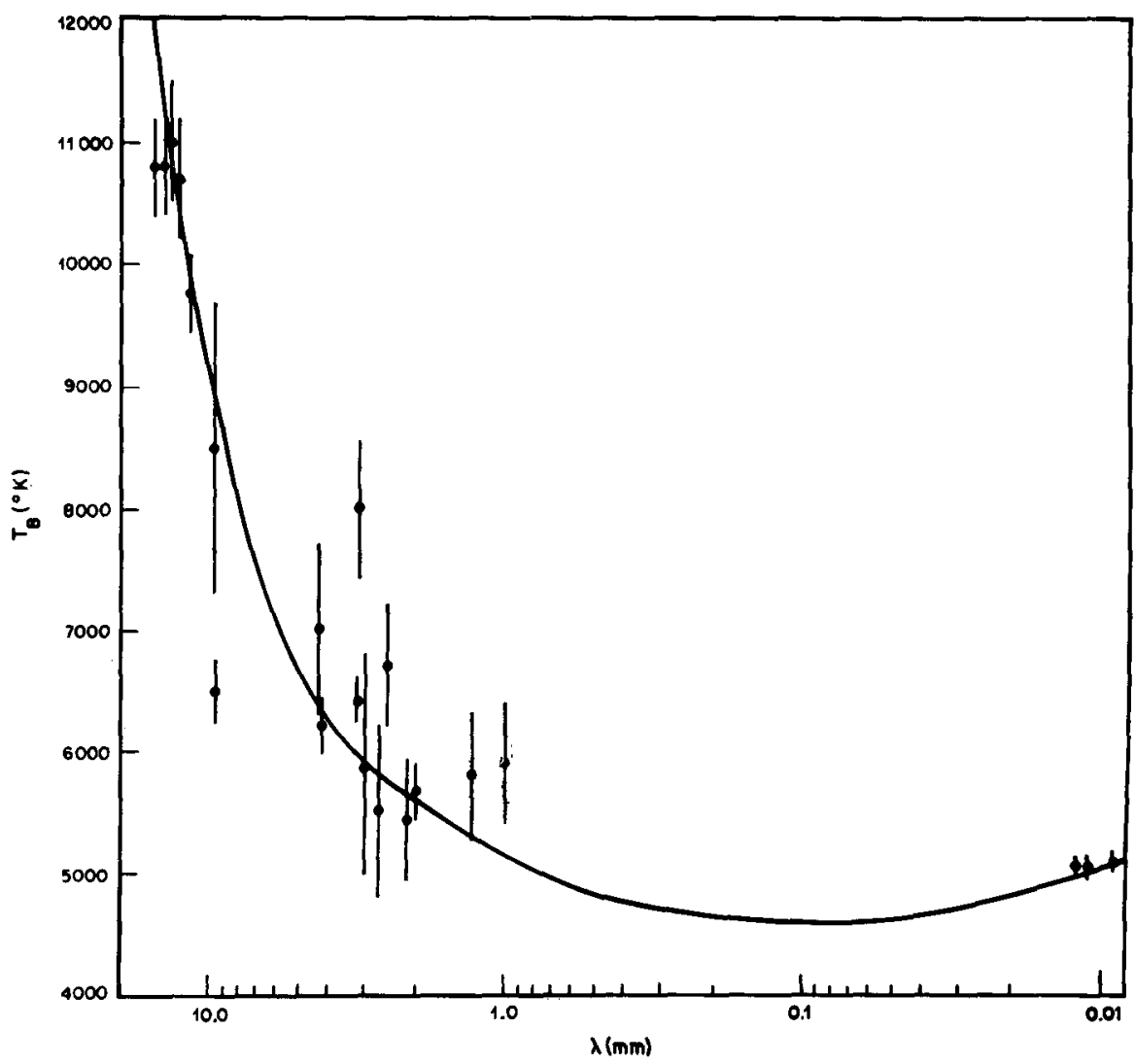

FIG. 3. Brightness temperature of the Sun plotted against wavelength.

Cepheus. In most directions the ratio of selective to total extinction, $R$, has a value around 3 to $3 \cdot 5$. Here, however, the value is close to 5 , presumably because of interstellar grains which are gray between 3000 and $10000 \AA$. As infrared techniques are developed it should become possible to deal directly with this problem by studying the thermal emission of interstellar grains over a wide range of wavelengths.

As Figure 5 shows, the interstellar medium becomes transparent in the infrared, revealing the obscured portions of the Galaxy. One of the most dramatic examples of the importance of this fact is the recent discovery of the galactic nucleus in the infrared by Becklin and Neugebauer. Figure 6 is taken from their paper, which is still in press. It shows the striking similarity between the nucleus of our Galaxy and that of M31. The total visual extinction in the path to the galactic centre is evidently about 25 magnitudes. The position found for the infrared centre agrees with the radio position of the source Sagittarius A.

The existence of infrared stars has been established since Hetzler's (1937) photographic work in the 1930's. The recent sky survey at 2.2 microns carried out by the 


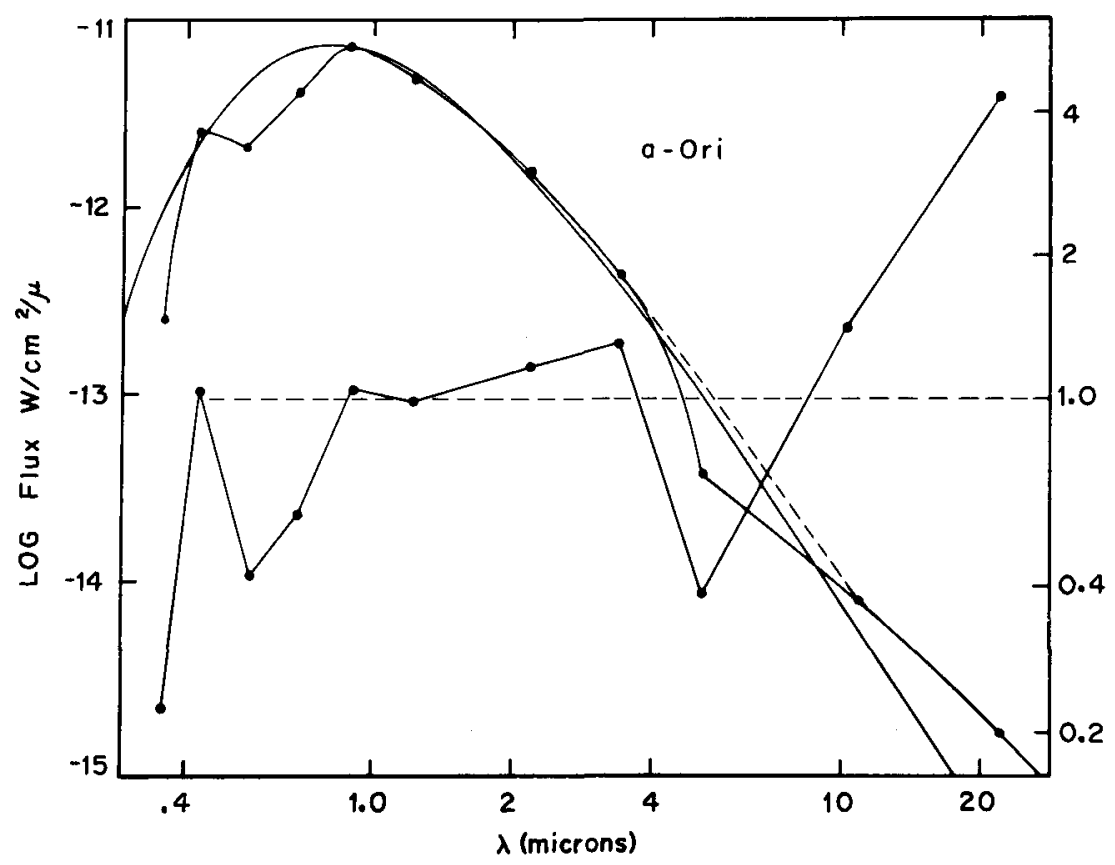

FIG. 4. Absolute spectral energy distribution of $\alpha$ Orionis showing a large excess at the longest wavelengths.

Caltech group (Neugebauer et al., 1965; Ulrich et al., 1966) is complete down to K magnitude of +3 and contains 5500 objects. Some of the more interesting of these stars have been studied in detail by various workers at Caltech (McCammon $e t$ al., 1967), Berkeley (Wing et al., 1967), Arizona (Johnson et al., 1965) and Rice (Low and Smith, 1966). Most of the objects are cool stars $\left(T_{\mathrm{e}}>1200^{\circ} \mathrm{K}\right)$ or are stars highly reddened by interstellar dust or by a circumstellar envelope (Wisniewski et al., 1967). Other infrared stars have been found by Mendoza (1966) of the University of Mexico while visiting Arizona. Studying various types of cool stars - some found photographically by Haro and Chivara - he discovered large infrared excesses in TTauri stars. Becklin and Neugebauer (1967) discovered a point source in Orion too faint to be seen by the sky survey, and this in turn led Kleinman and Low (1967) to the discovery at 22 microns of the first purely infrared nebula. Of chief interest is the question. How are these objects related to stellar or planetary formation? Poveda (1965), among others, had already suggested several possibilities. Figure 7 shows the result found by Low and Smith (1966) for R Monocerotis. Here it is not possible to explain the intense infrared continuum by extinction alone. A model was constructed which accounts for the high infrared luminosity and extended continuum as a protostar heating a preplanetary envelope of dust grains which reradiate at very long wavelengths. Figure 8 shows normalized spectral energy curves for five infrared objects; 


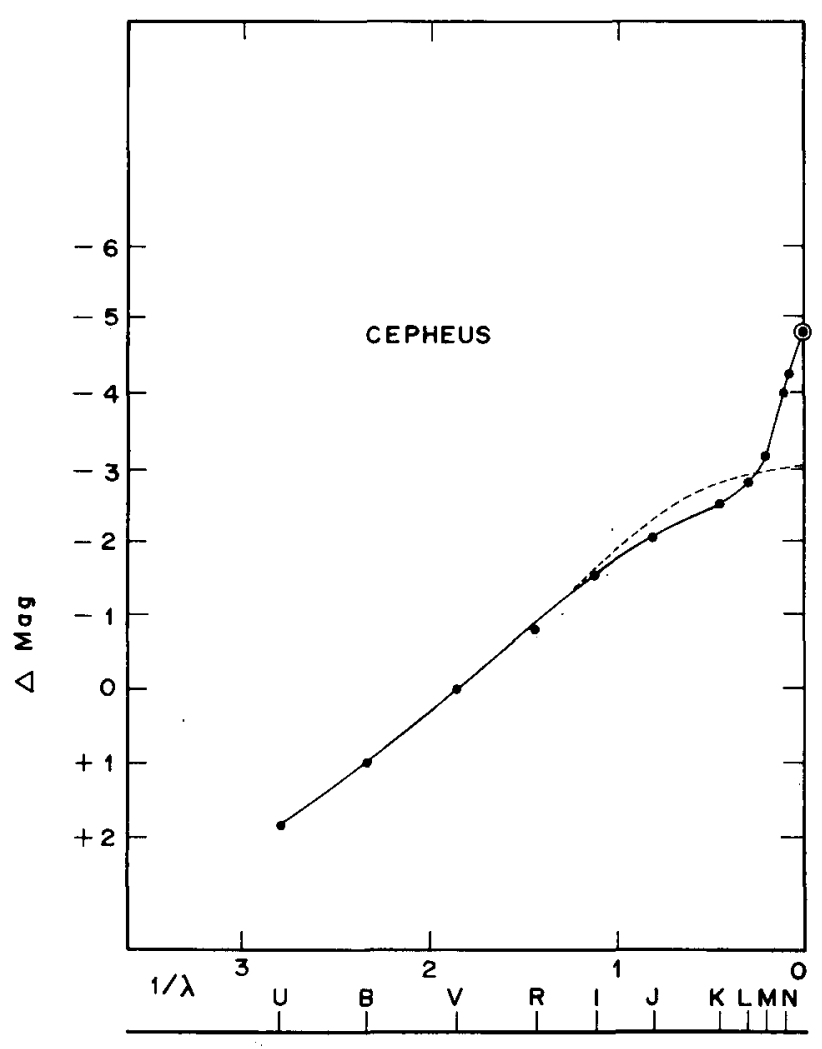

FIG. 5. Interstellar reddening law in Cepheus.

only one, NML Cygnus, appears to be unrelated to the problem of stellar formation. Figure 9 shows the size and position of the newly discovered infrared nebula in Orion, which may be a contracting cloud heated by a cluster of stars forming near its centre (Cameron, 1967; Hartmann, 1967).

The Crab Nebula presents a unique opportunity to study the synchrotron mechanism over an enormous spectral range. Moroz (1964) at the Crimean Observatory was the first to observe the Crab in the near infrared. Figure 10 shows our present knowledge of the spectrum; it includes new data obtained by Becklin at Caltech and Kleinmann at Rice.

The infrared properties of quasars are discussed later at this conference. Here we will only refer to the spectrum of $3 \mathrm{C} 273$ (Figure 11), point out that at 1.6 microns we have found it to be highly polarized, and take note of the new work at 1.6 and 2.2 microns on some 36 different quasars (Table 2).

Johnson's (1966) observations of 12 galaxies out to 3.4 microns produced a mean spectral energy distribution which can be explained on the basis of a suitable stellar population. This is not the case for the Seyfert galaxy NGC1068 observed by Pachol- 


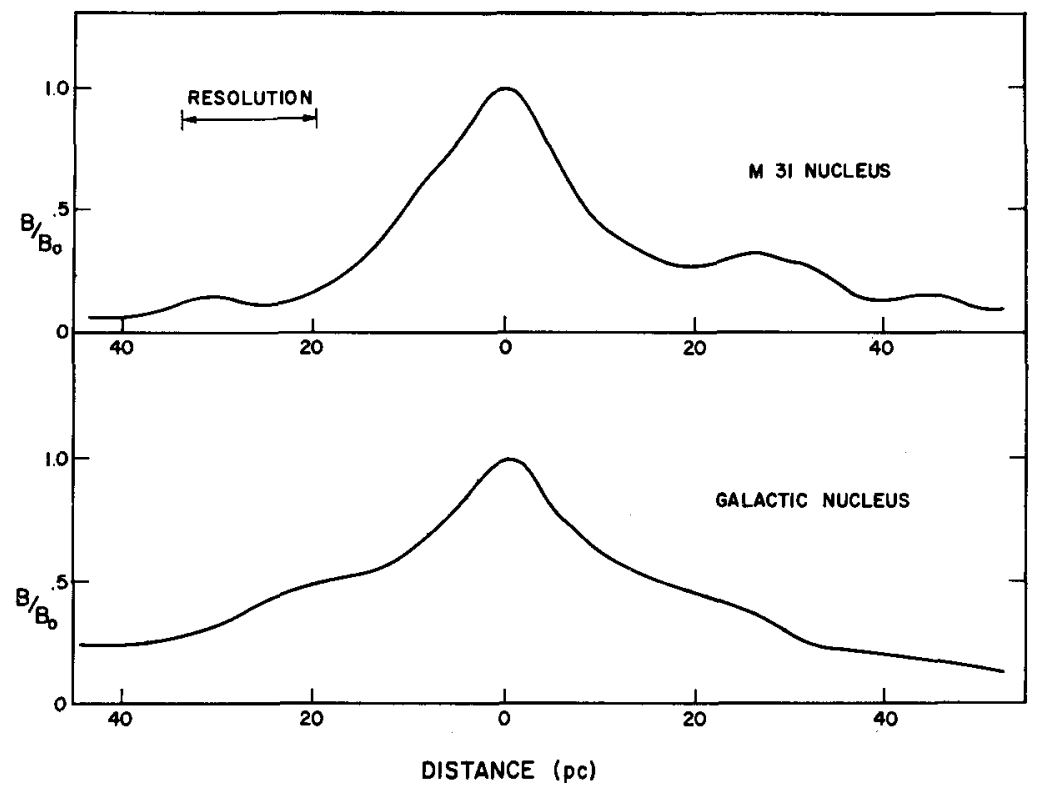

FIG. 6. A right ascension scan of M31 at $2 \cdot 2 \mu$ (Neugebauer and Becklin) is compared with a $2 \cdot 2 \mu$ scan of the centre of the galaxy. The latter was taken along a line $50^{\circ}$ to the galactic plane with a resolution degraded to the observation of $M 31$.

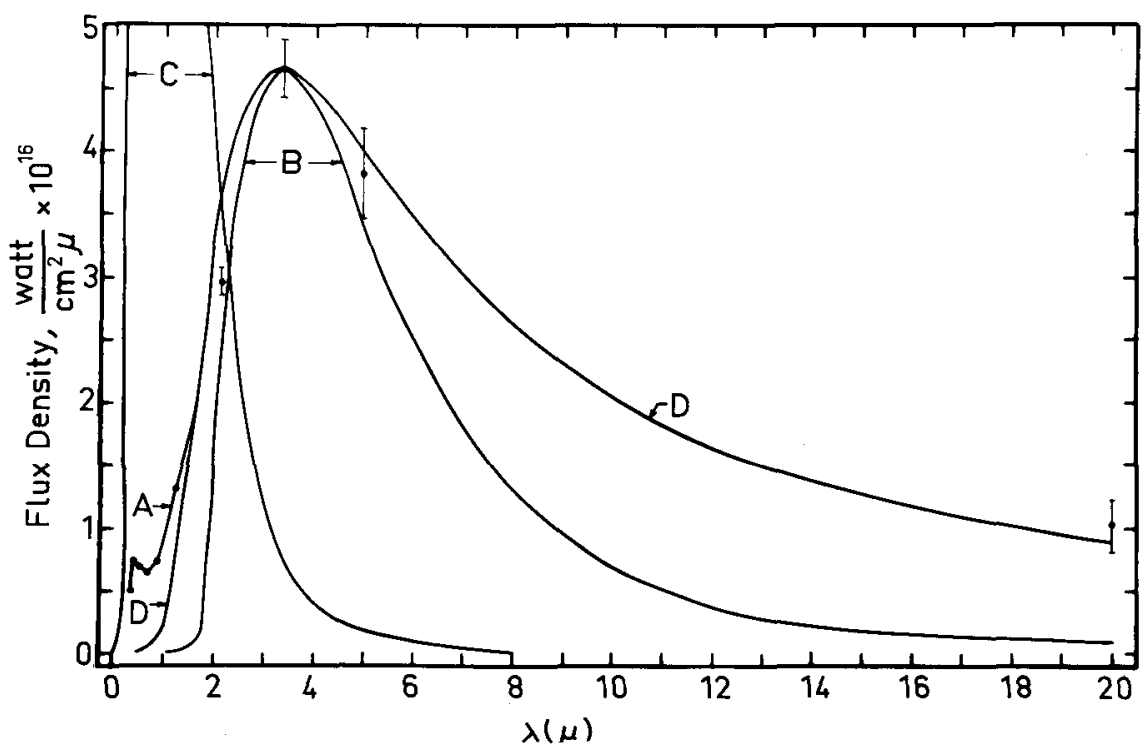

FIG. 7. Absolute spectral energy distribution of RMonocerotis compared to preplanetary dust model of Low and Smith. 


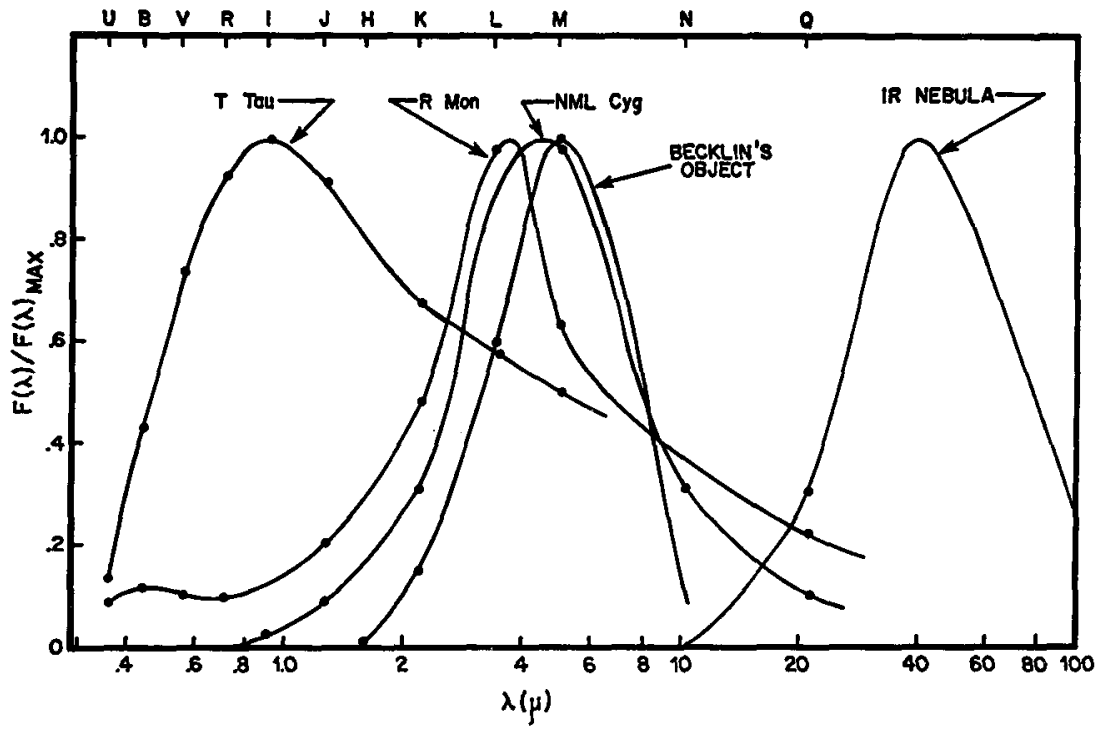

FIG. 8. Normalized spectral energy curves for 5 infrared objects.

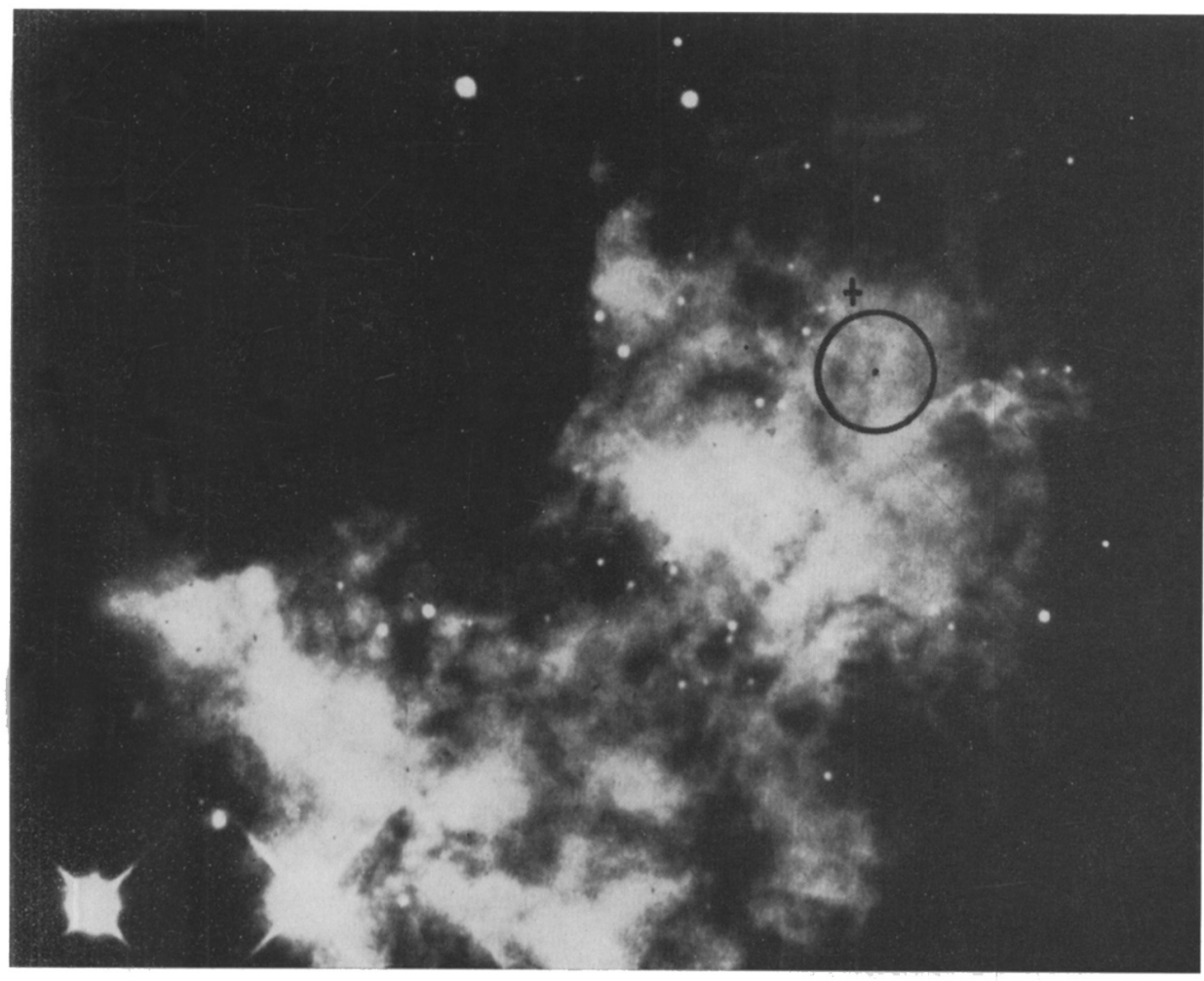

FIG. 9. Central region of Orion Nebula showing size and location of Infrared Nebula. 


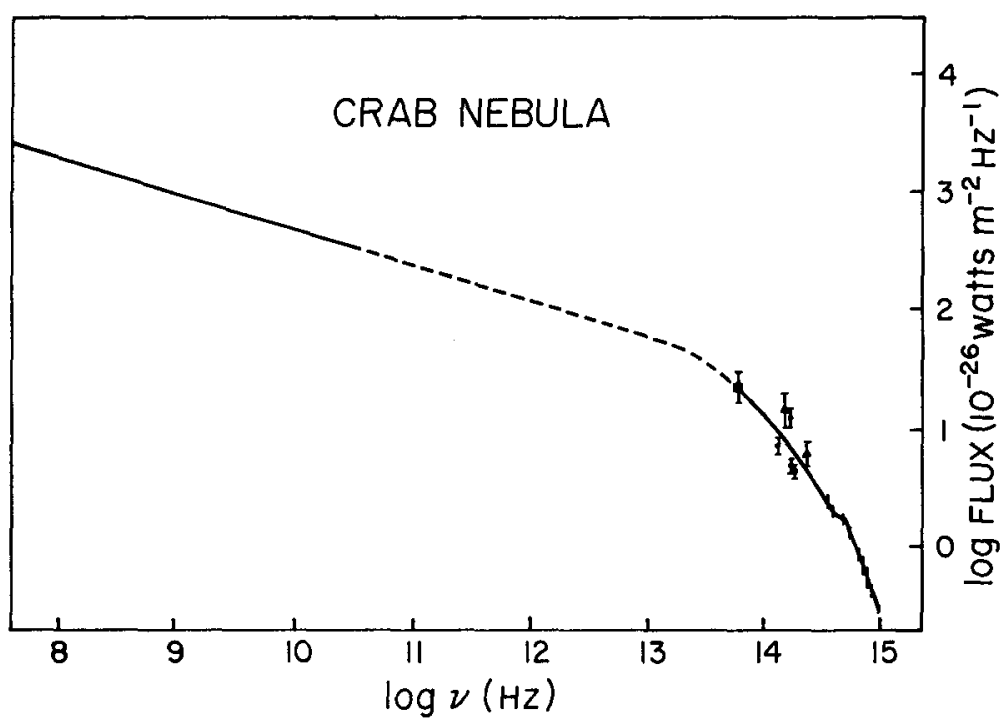

Fig. 10. $\log S(v)$ vs. $\log v$ for the Crab Nebula. Triangles are data from Moroz, filled circles from Becklin, and filled squares from Kleinmann.

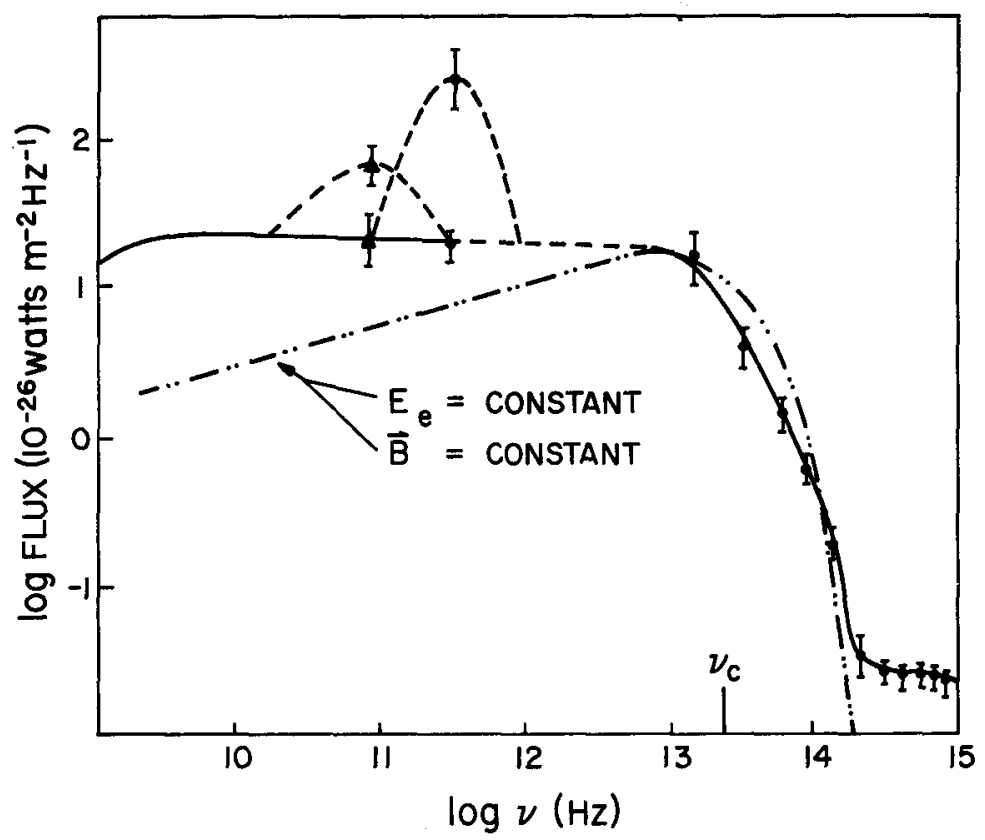

Fig. 11. $\log S(v)$ vs. $\log v$ for $3 C 273$, showing fat optical continuum, flat quiescent radio continuum, millimeter-wave outbursts and steep IR component. For comparison, the spectrum of monoenergetic relativistic electrons in a homogeneous magnetic field is plotted for an appropriate choice of critical frequency, $3 \times 10^{13} \mathrm{cps}$. Data at $10^{11} \mathrm{cps}$ due to Epstein. 
Table 2

Infrared properties of quasars at $\mathbf{1 . 6}$ and $\mathbf{2 . 2}$ microns

Designation

PHL 658

$3 \mathrm{C} 9$

*3C 48

PKS 0405-12

PKS 0859-14

TON 469

*3C 273

*PKS $1354+19$

*3C 298

*3C 309.1

*PKS 1510-08

*3C 323.1

*TON 256

*3C 334
$1.6 \mu$ Flux

$10^{-29} \mathrm{~W} / \mathrm{m}^{2} / \mathrm{Hz}$

$\begin{array}{cc}2.3 & \text { *3C } 345 \\ 0.43 & \text { *3C } 351 \\ 3.8 & \text { 3C } 371 \\ 3.3 & \text { *3C } 380 \\ \leqslant 2.5 & \text { 3C } 403 \mathrm{G} \\ 4 \cdot 6 & \text { 3C 403S } \\ 30 & \text { *PKS 2115-30 } \\ 0.80 & \text { 3C } 432 \\ 1.0 & \text { PKS } 2135-14 \\ 0.98 & \text { 3C } 446 \\ 1.1 & \text { *CT } 102 \\ 2.6 & \text { 3C } 454 \\ 3.8 & \text { *3C } 454 \cdot 3 \\ 1.4 & \end{array}$

$1.6 \mu$ Flux $10^{-29} \mathrm{~W} / \mathrm{m}^{2} / \mathrm{Hz}$

8.9

$3 \cdot 6$

16

$1 \cdot 0$

$2 \cdot 6$

28

$1 \cdot 1$

0.45

$3 \cdot 6$

2.9

$1 \cdot 1$

$0 \cdot 31$

0.74

* $2.2 \mu$ obs. by Neugebauer.

czyk and Wisniewski (1967) with the same apparatus. Figure 12 shows the spectrum of NGC 1068 including 5, 10 and 22 micron observations of Kleinmann and Low (previously unpublished) and the $3.4 \mathrm{~mm}$ result of Epstein (personal communication). Neither the optical spectrum nor the infrared spectrum can be produced by stars

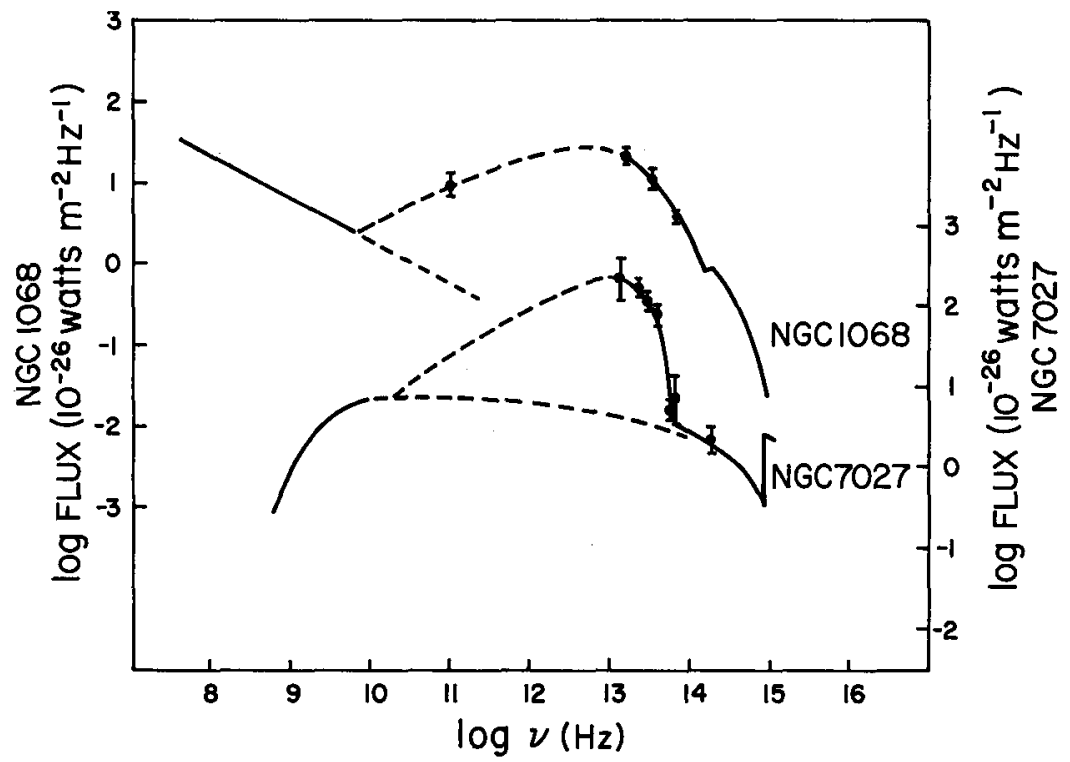

FIG. 12. $\log S(v) v s . \log v$ for the Seyfert galaxy NGC1068 and the planetary nebula NGC7027. Note the similarities between the infrared components in each object. Data at $10^{11}$ cps due to Epstein. 
alone. There is, however, some question whether to invoke a non-thermal process for the infrared, because the attendant strong radio emission is absent.

The planetary nebula, NGC7027, has the spectrum shown in Figure 12. Here the 10 micron data are from Gillett et al. (1967) and the 1·6, 5, and 22 micron data were recently obtained by the author, using the 200-inch telescope at Palomar Observatory. As is the case for Seyfert galaxies, the radio flux of the nebula is well below the infrared level. It seems plausible that the same mechanism is responsible for the infrared components of both types of object. Although much further work is required to prove this hypothesis, the simplest explanation is that the infrared is thermal reradiation from circumstellar or interstellar grains heated to only a few hundred degrees by a hot nearby source. As was shown in the case of R Monocerotis (Low and Smith, 1966), a large fraction of the available energy can be degraded into the infrared in this way.

In conclusion, it can be stated that infrared astronomy, until recently reserved for those interested in the planets, is becoming a major contributor to the rapidly expanding science of space astronomy.

\section{References}

Baldock, R.V., Bastin, J.A., Clegg, P.E., Emery, R., Gaitskell, J.N., Gear, A.E. (1965) Astrophys. J., 141, 1289.

Becklin, E.E., Neugebauer, G. (1967) Astrophys. J., 147, 799.

Becklin, E.E., Westphal, J. A. (1966) Astrophys. J., 145, 445.

Cameron, A. G.W. (1967) Infrared Conf., London, February.

Connes, P., Connes, J., Benedicts, W.S., Kaplan, L.D. (1967) Astrophys. J., 147, 1230.

Fedoseev, L.N. (1963) Radiofizika, 4, 425.

Gillett, F.C., Low, F.J., Stein, W. A. (1967) Astrophys. J., 149, L97.

Hartmann, W.K. (1967) Astrophys. J., 149, L87.

Hetzler, C. (1937) Astrophys. J., 86, 509.

Hoffman, W.F., Woolf, N.J., Frederick, C.L., Low, F.J. (1967) Science, 157, No. 3785, 187.

Johnson, H. L. (1965a) Comm. Lunar and Planetary Lab., No. 53.

Johnson, H.L. (1965b) Astrophys. J., 141, 923.

Johnson, H.L. (1966a) A. Rev. Astr. Astrophys., 4.

Johnson, H.L. (1966b) Astrophys. J., 143, 187.

Johnson, H.L. (1968) in Nebulae and Interstellar Matter. Ed. by B. M. Middlehurst and L.H. Aller, Univ. of Chicago (in press).

Johnson, H.L., Mitchell, R.I. (1963) Astrophys. J., 138, 302.

Johnson, H.L., Low, F.J., Steinmetz, D. (1965) Astrophys. J., 142, 808.

Johnson, H.L., Mitchell, R.I., Iriarte, B., Wisniewski, W.Z. (1966) Comm. Lunar and Planetary Lab., No. 63.

Kleinmann, D.E., Low, F.J. (1967) Astrophys. J., 149, L1.

Low, F.J. (1966) Astrophys. J., 146, 326.

Low, F.J., Davidson, A.W. (1965) Astrophys. J., 142, 1278.

Low, F. J., Gillespie, C.M. (1968) (in preparation).

Low, F.J., Johnson, H.L. (1964) Astrophys. J., 139, 1130.

Low, F. J., Smith, B. J. (1966) Nature, 212, 675.

McCammon, D., Munch, G., Neugebauer, G. (1967) Astrophys. J., 147, 575.

Mendoza, E.E. (1966) Astrophys. J., 143, 1010.

Moroz, V.I. (1964) Astrophys. J., 7, 755.

Neugebauer, G., Martz, D.E., Leighton, R.B. (1965) Astrophys. J., 142, 399. 
Pacholczyk, A.G., Wisniewski, W.Z.(1967) Astrophys. J., 147, 394.

Poveda, A. (1965) Bol. Obs. Tonantzintla y Tacubaya, 4, 15; ibid., p. 22.

Ulrich, B.T., Neugebauer, G., McCammon, D., Leighton, R.B., Hughes, E.E., Becklin, E. (1966) Astrophys. J., 146, 288.

Westphal, J.A., Wildey, R. L., Murray, B.C. (1965) Astrophys. J., 142, 799.

Wing, R.F., Spinrad, H., Kuhi, L.V. (1967) Astrophys. J., 147, 117.

Wisniewski, W.Z., Wing, R. F., Spinrad, H., Johnson, H.L. (1967) Astrophys. J., 148, L29.

\section{DISCUSSION}

$Y$. Ohman: It would be extremely interesting to hear if one could make polarization measurements of the thermal emission from grains. We found some years ago that the thermal emission of the very narrow metallic filaments are strongly polarized when the filaments are of a size comparable to the wavelength of light. It might be different in the infrared but still this question might still be worth looking into.

F.J. Low: What 1 referred to was the possibility of observing in the far infrared beyond 25 microns. These observations will give an entirely new handle on the problem of interstellar grains. The condition that you just mentioned would not be met there. The measurements in the infrared are quite possible and I think will become very important in the future.

G.P. Kuiper: You did not say in what wavelength the measurements at the galactic centre were made.

F.J. Low: The measurements were made at a variety of wavelengths, $1 \cdot 6,2 \cdot 2$, and $3.4 \mu \mathrm{m}$. They have not yet succeeded in making measurements at $5 \mu \mathrm{m}$, although there is no doubt it can be measured. Because the galactic centre is an extended source, about $1 \mathrm{~min}$ of arc in size, it will be difficult to measure (at longer wavelengths) from the ground. At wavelengths shorter than 1.2 microns no radiation has been detected, and only an upper limit has been determined.

J.A. Roberts: I would like to make an appeal for standardization of the flux units in watts per square meter per micron rather than the standard watts per square meter per unit frequency which is used at radio frequencies. I think it is quite important, since it changes the shape of the curves completely.

F.J. Low: We have unhappily used two systems of units for flux in the infrared, because we lie between the radio and the optical astronomers. The radio astronomers like watts per square meter per Hertz, but it is also useful at times to use watts per square centimeter per micron.

$Z$. Turto: Do you have any information on the radiation from the Hin region in the Orion nebula?

F.J. Low: There are quite a number of results on $\mathrm{HII}$ regions which are new. Our measurements on the Orion nebula, that is the HII region and not the infrared nebula which I showed, indicate that the surface brightness is about 10 times higher than the free-free continuum would predict. This is the case in 7027 , the planetary nebula. So Hir regions in the infrared are going to be very interesting.

S. Plagemann: In your measurements of T Tauri and R Monocerotis, what considerations have you made for the fact that these are variable stars? All your measurements have been spaced very close to each other or done over several lines.

F.J. Low: The observations have been carried out over several months. We have not been able to relate optical variations to infrared variations if indeed, infrared variations do exist. This general subject is one we hope can be explored in the future, and will give a better understanding of the rather complex models. 